\title{
Coletar morcegos por seis ou doze horas a cada noite?
}

\author{
Carlos E. L. Esbérard \& Helena G. Bergallo
}

Departamento de Ecologia, IBRAG, Universidade do Estado do Rio de Janeiro. Rua São Francisco Xavier 524, 20559-900 Rio de Janeiro, Rio de Janeiro, Brasil. E-mail: cesberard@terra.com.br

\begin{abstract}
Sampling bats for six or twelve hours in each night? Brazilian researchers of bats concentrate the sampling activity with mist nets in the first half of the night, when it is described that concentrates most of the activity of the species. To evaluate the results of the total captured and species richness during the night, we analyzed the time of capture in inventories developed in southeastern Brazil. The total sampling comprised 570 captures and 25 species of three different families. Of the 18 species in that was possible to calculate the average of time of capture, only two insectivore species showed values previous to 21:00 h. We noticed high similarity in the abundance of the species between the first and the second half of the night. The all night sampling can result in larger richness and should be the chosen methods for inventory.
\end{abstract}

KEY WORDS. Activity, methods, richness, sampling.

RESUMO. Pesquisadores brasileiros de morcegos concentram a atividade de coleta com redes de neblina na primeira metade da noite, onde é descrito que ocorre a maior parte da atividade das espécies a serem coletadas. Para avaliar os resultados quanto ao total capturado e a riqueza de espécies em parte da noite, analisamos o horário de captura em inventário realizado no sudeste do Brasil. A amostragem total compreendeu 570 capturas e 25 espécies de três diferentes famílias. Das 18 espécies em que foi possível calcular a média do horário de capturas, apenas duas espécies insetívoras aéreas apresentaram valores anteriores às 21:00 h. Notou-se grande similaridade quanto a abundância das espécies, tanto na primeira metade da noite quanto na segunda metade. A riqueza e o total capturado resultaram em elevada similaridade entre a amostragem observada do pôr-do-sol às 00:00 e das 00:01 h ao amanhecer. A realização de coletas durante toda a noite pode resultar em maior riqueza e deve ser o método escolhido para um inventário.

PALAVRAS CHAVE. Amostragem, atividade, métodos, riqueza.

A maioria dos estudos desenvolvidos com morcegos concentra as coletas com redes de neblina nas primeiras horas da noite após o pôr-do-sol. É consenso entre os pesquisadores que as primeiras horas após o pôr-do-sol são as mais produtivas, com a taxa de captura declinando três a seis horas após (Jones et al. 1996, Marinho-Filho \& Sazima 1989, Pedro \& Taddei 2002, Aguiar \& Marinho-Filho 2004). Na maioria absoluta das espécies de morcegos estudadas até o momento, a atividade noturna é sincronizada com o pôr-do-Sol (ChURCH 1957, HerReID \& DAvis 1966, Bateman \& Vaughan 1974, Erkert 1978, 1982, Avery 1986, MCANEY \& Fairley 1988, IsaAC \& Marithmu 1993, CatTo et al. 1995, Kunz $\&$ Anthony 1996). A coleta por toda a noite tem a vantagem de amostrar todo o período de atividade das espécies de morcegos, mas resulta em grande desgaste físico, que é multiplicado em procedimentos que necessitam de noites consecutivas de trabalho. Assim, coletas que duram até 6 h após o pôr-do-sol, supostamente cobrem o maior período de atividade dos morcegos, priorizando os momentos de maior produtividade. Contudo, o efeito desta metodologia na riqueza e composição das espécies permanece desconhecido. Para avaliar os resultados quanto ao total capturado, à riqueza de espécies e a composição de espécies, analisamos o horário de captura em diferentes períodos da noite.

\section{MATERIAL E MÉTODOS}

As coletas foram realizadas no Açude da Solidão, Parque Nacional da Tijuca, Município do Rio de Janeiro (2257'40,6"S e $043^{\circ} 17^{\prime} 61,0^{\prime \prime} \mathrm{W}, 535 \mathrm{~m}$ de altitude) entre 1995 e 1997. Para uma melhor caracterização da área veja EsBÉRARD (2003). As redes foram armadas em trilhas já existentes e sobre o espelho d'água, permanecendo abertas por toda a noite. Um total de 15 noites de coleta ( $610 \mathrm{~h}$ de esforço) foi realizado, independente de qualquer fase do ciclo lunar (média de $60,3 \pm 35,7 \%$ da face iluminada da lua). A cada noite de 8 a 10 redes $(7 \times 2,5$ $\mathrm{m})$ foram armadas. Os morcegos capturados foram marcados com tatuagem no dactilopatágio e as recapturas observadas na mesma noite não foram consideradas na análise. O horário de captura foi considerado independente do horário de verão ado- 
tado. Os horários de captura ao longo da noite foram separados em dois grupos, aqueles do pôr-do-sol até às 00:00 h e de 00:01 h até uma hora após o amanhecer. Para analisar a variação da média de capturas por noite transformamos o horário de captura em minutos após o pôr-do-sol, usando as Efemérides Astronômicas (Observatório Nacional, Rio de Janeiro, CNPq).

A similaridade entre as abundâncias de captura para as espécies nas duas metades da noite foi testada usando o teste Mann-Whitney. A similaridade das espécies das duas metades da noite foi testada usando o Coeficiente de Jaccard. Nós testamos à hipótese de que a quantidade de capturas é diferente nas duas metades da noite usando o teste de Qui-quadrado. Todas as análises foram feitas no programa Systat.

\section{RESULTADOS}

A amostragem total compreendeu 570 capturas de 25 espécies de três diferentes famílias: Phyllostomidae (13 gêneros e 18 espécies), Vespertilionidae (quatro gêneros e cinco espécies) e Molossidae (dois gêneros e duas espécies) (Tab. I). Das 18 espécies em que foi possível calcular a média do horário de capturas, apenas duas espécies (10,53\%) apresentaram valores anteriores às 21:00 h-Molossus molossus (Pallas, 1766) e Eptesicus brasiliensis (Desmarest, 1819). Estas mesmas espécies foram também as que apresentaram as menores taxas de captura após as 00:01 h. Nove espécies apresentaram uma média de horário de captura antes das 00:00 h e outras nove após as 00:01 h. Vinte e duas espécies apresentaram horário mínimo de captura antes das 00:00 h e apenas três depois das 00:01 h (Tab. I).

Analisando a comunidade obtida nos dois períodos de tempo estudados nota-se grande similaridade quanto à abundância das espécies $(\mathrm{U}=0,001 \mathrm{p}=1,00)$. A riqueza e o total capturado são similares entre o total analisado do pôr-do-sol à 00:00 h e da 00: $01 \mathrm{~h}$ ao amanhecer. As duas metades da noite também são similares com a riqueza de toda a noite (Tab. II).

A média do horário de capturas apresentou relação linear positiva $(r=0,80, p<0,001)$ com a percentagem de capturas

Tabela I. Horário de capturas para as 25 espécies analisadas no Açude da Solidão, com o horário mínimo, máximo, média, desvio padrão (SD), total de capturas (N) e percentagem de capturas obtidas depois de 00:01 h.

\begin{tabular}{|c|c|c|c|c|c|c|c|}
\hline \multirow{2}{*}{ Espécies } & \multirow{2}{*}{ Sigla } & \multicolumn{5}{|c|}{ Horário de capturas (h) } & \multirow{2}{*}{$\begin{aligned} & \text { Capturas } \\
> & 00: 01 \mathrm{~h}(\%)\end{aligned}$} \\
\hline & & Mínimo & Máximo & Média & SD & $\mathrm{N}$ & \\
\hline Anoura caudifera (E. Geoffroy, 1818) & Anca & $17: 37$ & $06: 15$ & $00: 32$ & 5,69 & 5 & 40,00 \\
\hline Myotis ruber (E. Geoffroy, 1806) & Myru & $17: 48$ & 02:00 & $22: 14$ & & 2 & 50,00 \\
\hline Myotis nigricans (Schinz, 1821) & Myni & $17: 48$ & $06: 30$ & $23: 33$ & 4,56 & 19 & 26,32 \\
\hline Carollia perspicillata (Linnaeus, 1758) & Cape & $18: 00$ & $06: 20$ & $23: 26$ & 3,67 & 32 & 34,38 \\
\hline Artibeus lituratus (Olfers, 1818) & Arli & $18: 00$ & $06: 00$ & $00: 17$ & 2,78 & 211 & 49,76 \\
\hline Artibeus fimbriatus Gray, 1838 & Arfi & $18: 00$ & 07:00 & $00: 31$ & 3,18 & 113 & 52,21 \\
\hline Molossus molossus (Pallas, 1766) & Momo & $18: 10$ & $00: 30$ & 19:11 & 1,22 & 25 & 4,00 \\
\hline Eptesicus brasiliensis (Desmarest, 1819) & Epbr & $18: 12$ & 00:00 & 19:09 & 1,65 & 3 & 0,00 \\
\hline Sturnira lilium (E. Geoffroy, 1810) & Stli & $18: 15$ & 06:00 & $00: 23$ & 4,29 & 41 & 48,78 \\
\hline Tonatia bidens (Spix, 1823) & Tobi & $18: 30$ & $03: 34$ & $23: 10$ & 3,75 & 6 & 33,33 \\
\hline Vampyressa pusilla (Wagner, 1843) & Vapu & $18: 40$ & 02:15 & $21: 34$ & 2,92 & 7 & 14,29 \\
\hline Micronycteris megalotis (Gray, 1842) & Mime & $18: 50$ & - & - & - & 1 & 0 \\
\hline Artibeus cf jamaicensis Leach, 1821 & Arja & 19:00 & $06: 45$ & $23: 36$ & 3,19 & 37 & 45,95 \\
\hline Pygoderma bilabiatum (Wagner, 1843) & Pybi & 19:05 & $01: 35$ & $22: 20$ & & 2 & 50,00 \\
\hline Artibeus obscurus Schinz, 1821 & Arob & 19:15 & $05: 30$ & 00:07 & 2,78 & 44 & 52,27 \\
\hline Platyrrhinus lineatus (E. Geoffroy, 1810) & Plli & 19:15 & $06: 15$ & 01:18 & 4,65 & 4 & 75,00 \\
\hline Diphylla ecaudata (Spix, 1823) & Diec & $19: 45$ & $04: 30$ & 00:04 & 4,88 & 4 & 50,00 \\
\hline Chiroderma doriae Thomas, 1891 & Chdo & $20: 50$ & 04:20 & 01:10 & 2,84 & 6 & 66,67 \\
\hline Desmodus rotundus (E. Geoffroy, 1810) & Dero & $21: 04$ & - & - & - & 1 & 0 \\
\hline Chiroderma villosum Peters, 1860 & Chvi & 22:00 & - & - & - & 1 & 0 \\
\hline Histiotus velatus (I. Geoffroy, 1824) & Hive & $22: 17$ & 04:50 & $01: 34$ & - & 2 & 50,00 \\
\hline Lonchophylla mordax Thomas, 1903 & Lomo & $23: 30$ & - & - & - & 1 & 0 \\
\hline Lasiurus borealis (Muller, 1776) & Labo & $02: 15$ & - & - & - & 1 & 100 \\
\hline Artibeus cinereus (Gervais, 1856) & Arci & $02: 40$ & - & - & - & 1 & 100 \\
\hline Eumops auripendulus (Schaw, 1800) & Euau & $05: 14$ & - & - & - & 1 & 100 \\
\hline
\end{tabular}



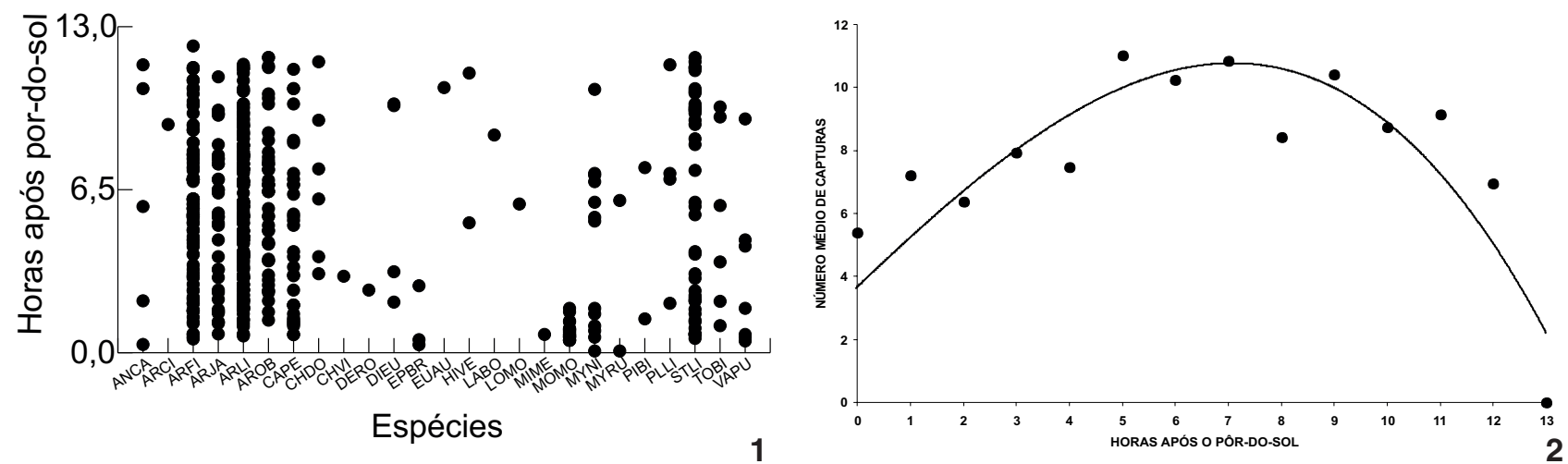

Figuras 1-2. (1) Capturas das espécies expressas por minutos após o pôr-do-sol (veja as siglas das espécies na tabela I); (2) variação da média de capturas por hora a partir do pôr-do-sol.

Tabela II. Riqueza, total de capturas e similaridade de Jaccard para os períodos compreendidos do pôr-do-sol à 00:00 h, da 00:01 h até o amanhecer e para toda a noite.

\begin{tabular}{lccc}
\hline & \multicolumn{3}{c}{ Períodos analisados } \\
\cline { 2 - 4 } & $<00: 00 \mathrm{~h}$ & $>00: 01 \mathrm{~h}$ & Noite inteira \\
\hline Riqueza & 22 & 20 & 25 \\
Capturas & $308(54,03 \%)$ & $262(45,97 \%)$ & 570 \\
Similaridade & 0,88 & 0,80 & - \\
\hline
\end{tabular}

após as 00h01min, isto é, quanto mais elevada a média do horário, mais capturas foram observadas após as 00h01min.

A hipótese de que a quantidade de capturas não difere nas duas metades da noite é verdadeira $\left(\chi^{2}=3,71, \mathrm{df}=1,0,10>\mathrm{p}>0,05\right)$ (Fig. 1). A abundância média de capturas mostrou seu pico cinco horas após o pôr-do-sol e manteve-se alta por toda a noite (Fig. 2).

\section{DISCUSSÃO}

Apenas duas espécies apresentaram média do horário de captura antes das 21:00 h, ambas as espécies com hábito alimentar insetívoro aéreo - M. molossus e E. brasiliensis (KaLKo et al. 1996). Estas espécies apresentam atividade diária bimodal, com pico de curta duração iniciado durante ou logo após o pôr-do-sol e, eventualmente, com um segundo pico, menor, próximo ao amanhecer, quando observa-se a maior disponibilidade de insetos (Marques 1986, Fenton et al. 1998). O reduzido período de atividade e a predominância da atividade próxima ao crepúsculo podem explicar o menor valor encontrado na média do horário de captura destas espécies.

A maioria absoluta de espécies de morcegos inicia sua atividade imediatamente antes, durante ou logo após o pôr-do-sol (ERKert 1978, Avery 1986, Catto et al. 1995). É descrito que espécies frugívoras e hematófagas têm sua atividade reduzida no meio da noite como forma de minimizar o risco de predação, podendo apresentar padrão bimodal, com um maior pico próximo ao pôr-do-sol (Fleming 1988) ou, como observado em Artibeus lituratus, maior pico próximo ao amanhecer (BERNARD 2002). A relação entre a média do horário de captura e o total capturado após as 00:01 h demonstra que a atividade de espécies, principalmente as não insetívoras, pode estender-se por toda a noite.

Menor número de capturas poderia ser esperado na segunda metade da noite, pois Marinho-Filho \& SAzima (1989), Pedro \& Taddei (2002) e Aguiar \& Marinho-Filho (2004) encontraram um decréscimo do total de capturas três horas após o pôr-do-sol para algumas espécies de Phyllostomidae. Tal fato, não observado nesta análise, pode ser decorrente de terem sido realizadas coletas distribuídas em todo o ciclo lunar, enquanto os demais autores preferiram realizar coletas em determinado período do ciclo lunar. Nossos dados concordam com BERNARD (2002) que encontrou para morcegos frugívoros atividade por toda a noite.

A hipótese de que as duas metades da noite diferem quanto ao número de capturas foi descartada, demonstrando que para obter o máximo de capturas devem ser realizadas coletas por toda a noite. A média de capturas teve o máximo cinco horas após o pôr-do-sol e manteve-se alta (maior que 5,0 capturas/h) até a $12^{\mathrm{a}}$ hora após o pôr-do-sol sustentando ser produtiva a realização de coletas por toda a noite. A realização de amostragens durante toda a noite resultou em maior riqueza, no entanto, a captura de espécies após as 00:01 h deve ser encarada com cuidado, pois as espécies amostradas neste período, Artibeus cinereus (Gervais, 1856), Lasiurus borealis (Muller, 1766) e Eumops auripendulus (Schaw, 1800) foram representadas por uma única captura e são espécies que apresentam atividade também junto ao crepúsculo. Entretanto, estas espécies são pouco representadas em inventários realizados no sudeste do Brasil e a realização de coletas por toda a noite, que resultarão em maior número de capturas, pode incrementar a chance de amostrar as espécies mais raramente observadas.

Uma alternativa, menos onerosa quanto ao esforço de coleta por toda a noite, pode ser realizar coletas em noites consecutivas em diferentes horários, iniciando parte das coletas às 00:01 h. 


\section{AGRADECIMENTOS}

Agradecemos a Administração do Parque Nacional da Tijuca pela permissão de coleta (Processo 001418/95-21-SUPES/ RJ/IBAMA). Agradecemos as contribuições do Dr. Fernando C. Passos na versão final. C. Esbérard recebeu uma bolsa do CNPq (processo 152910/2004-0) e HGB recebeu bolsa de produtividade do CNPq (processo 301372/95-0).

\section{REFERÊNCIAS BIBLIOGRÁFICAS}

Aguiar, L.M.S. \& J. Marinho-Filho. 2004. Activity patterns of nine phyllostomid bat species in a fragment of the Atlantic Forest in southeastern Brazil. Revista Brasileira de Zoologia, Curitiba 21 (2): 385-390.

Avery, M.I. 1986. Factors affecting the emergence times of Pipistrelle bats. Journal of Zoology, London, 209: 293-296.

Bateman, G.C. \& T.A. Vaughan. 1974. Nightly activities of mormoopid bats. Journal of Mammalogy, Provo, 55 (1): 45-65.

BERNARD, E. 2002. Diet, activity and reproduction of bat species (Mammalia, Chiroptera) in central Amazonia, Brazil. Revista Brasileira de Zoologia, Curitiba, 19 (1): 173-188.

CatTo, C.M.C.; P.A. Racey \& P.J. STePhenson. 1995. Activity patterns of the serotine bat (Eptesicus serotinus) at a roost in southern England. Journal of Zoology, London, 235: 635-644.

Church, H. 1957. The time of emergence of Pipristelle. Zoological Society of London, London, 128: 606-608.

ERKERT, H.G. 1978. Sunset- related timing of flight activity in neotropical bats. Oecologica, Berlin, 37: 59-67.

ERKERT, H.G. 1982. Ecological aspects of bat activity rgythms, p. 201-242. In: T.H. Kunz (Ed.). Ecology of bats. New York, Plenum Press, 425p.

EsbéraRd, C.E.L. 2003. Diversidade de morcegos em uma área de Mata Atlântica regenerada no sudeste do Brasil (Mammalia: Chiroptera). Revista Brasileira de Zoociências, Juiz de Fora, 5 (2): 189-204.

Fenton, M.B.; I.L. Rautenbach; J. Rydell; H.T. Arita; J. Ortega; S. Boucahrd; M.D. Hovorka; B. Lim; E. Odgren; C.V. Portfors; W.M. Scully \& M.J. Vonhof. 1998. Emergence, echolocation, diet and foraging behavior of Molossus ater (Chiroptera: Molossidae). Biotropica, Storrs, 30 (2): 314-320.

FLEMING, T.H. 1978. The short-tailed fruit bat: A study in plantanimal interactions. Chicago, University of Chicago Press, $365 p$.

HerReID, C.F. II \& R.B. DAvis. 1966. Flight patterns of bats. Journal of Mammalogy, Provo, 40 (1): 140-150.

IsAaC, S.S. \& G. Marimuthu. 1993. Early outflying and late homeflying in the India pygmy bat under natural conditions. Oecologia, New York, 96: 426-430.

Jones, C.; W.J. MCsheA; M.J. ConRIY \& T.H. Kunz. 1996. Capturing mammals, p. 115-155. In D.E. Wilson; F. Russel Cole; J.D. Nichols; R. Rudran \& M.S. Foster (Eds). Measuring and monitoring biological diversity. Standard methods for mammals. Washington Smithsonian Intitution Press, 409p.

KalKo, E.K.V.; C.O. Handley JR. \& D.H. Handley. 1996. Organization, diversity and long-term dynamics of a neotropical bat community, p. 503-553. In: M.L. Cody \& J.A. SMALLWOOD (Eds). Long-term studies of vertebrate communities. San Diego, Academic Press, 597p.

Kunz, T.H. \& E.L.P. Anthony. 1996. Variation in the timing of nightly emergence behavior in the little brown bat, Myotis lucifugus (Chiroptera: Vespertilionidae), p. 225-235. In: Contribution in mammalogy: a memorial volume honoring Dr. J. Knox Jones Jr. Lubbock, Museum Texas Tech University press, $315 \mathrm{p}$.

Marinho-Filho, J. \& I. Sazima. 1989. Activity patterns of six phyllostomid bat species in Southeastern Brazil. Revista Brasileira de Biologia, São Carlos, 49: 777-782.

MARQUES, S.A. 1986. Activity cycle, feeding and reproduction of Molossus ater (Chiroptera: Molossidae) in Brazil. Boletim do Museu Paraense Emilio Goeldi, Belém, 2: 159-179.

Mcaney, C.M. \& J.S. Fairley. 1988. Activity patterns of the lesser horseshoe bat Rhinolophus hipposideros at summer roosts. Journal of Zoology, London, 216: 325-338.

Pedro, W.A. \& V.A. TAdDEI. 2002. Temporal distribution of five bat species (Chiroptera, Phyllostomidae) from Panga Reserve, south-eastern Brazil. Revista Brasileira de Zoologia, Curitiba, 19 (3): 951-954.

Recebido em 26.IV.2005; aceito em 11.XI.2005. 